\title{
Anti-mycobacterial treatment reduces high plasma levels of CXC-chemokines detected in active tuberculosis by cytometric bead array
}

\author{
Caroline de Souza Almeida, Clarice Abramo, Caio César de Souza Alves, Luciano Mazzoccoli, \\ Ana Paula Ferreira, Henrique Couto Teixeira/ ${ }^{+}$
}

Departmento de Parasitologia, Microbiologia e Imunologia, Instituto de Ciências Biológicas, Universidade Federal de Juiz de Fora, Juiz de Fora, MG, Brasil

Chemokines recruit and activate leukocytes, assisting granuloma formation. Herein, we evaluated plasma chemokines in patients with active tuberculosis (ATB) and after completing treatment (TTB) and compared them to $B C G$-vaccinated healthy controls (HC). Levels of chemokines were measured by cytometric bead array. Levels of CXCL8, CXCL9 and CXCL10 were higher in ATB patients compared to HC, but they decreased in TTB. Levels of CCL2 and CCL5 in ATB patients were similar to those observed in HC. Thus, the high levels of CXC-chemokines detected during ATB, which can modulate the trafficking of immune cells from the periphery to the site of infection, were reversed by anti-mycobacterial treatment.

Key words: tuberculosis - chemokines - cytometric bead array - chemotherapy

Immune control of tuberculosis (TB) is thought to be partly mediated by cytokines, such as IFN- $\gamma$ and TNF- $\alpha$ which modulate cellular immune responses and chemokine production. Chemokines are involved in chemoattraction, activation of leukocytes and facilitation of granuloma formation (Algood et al. 2003). Tuberculous granulomas consist of macrophages, dendritic cells, T and $B$ cells and fibroblasts surrounding cells infected with the bacteria and thus containing the dissemination and growth of the bacteria in the host. Production of CXCL8 during Mycobacterium tuberculosis infection modulates neutrophil and lymphocyte migration to the granuloma and high levels of CXCL8 have been associated with necrotising granulomas in infected lung tissue (Zhang et al. 1995). Other chemokines, such as CXCL9, CXCL10, CCL2 and CCL5, also act in the immune response against TB. Mice deficient in CXCR3, a receptor for CXCL9 and CXCL10, showed altered and delayed granuloma formation after infection with M. tuberculosis (Seiler et al. 2003). CCL2 and CCL5 are produced primarily by macrophages and are involved in cellular trafficking in TB (Algood et al. 2003). CCL2-deficient mice had higher mycobacterial burdens in the lung and spleen after three weeks of $M$. tuberculosis infection, but they eventually controlled the infection ( $\mathrm{Lu}$ et al. 1998). A recent study using the CBA methodology showed significant increases in CXCL8, CXCL9, CXCL10 and CCL5 in the plasma of pulmonary TB patients in comparison to healthy subjects (Pokkali \& Das 2009). They also demonstrated that the receptors CXCR3 and CCR5

Financial support: CNPq (310912/2006-7), FAPEMIG (CBB 1464/06, CBB PPM 0247/08), WOTRO, CAPES

+ Corresponding author: henrique.teixeira@pq.cnpq.br

Received 13 February 2009

Accepted 29 September 2009 were up-regulated in T helper (Th) cells, while CXCR2 was up-regulated in Th cells, natural killer cells and neutrophils. The current knowledge of the effects of anti-TB chemotherapy on blood chemokine levels in TB patients is limited. Chemokine expression has been characterised by conventional techniques, such as PCR and ELISA, to assess gene expression and protein levels, respectively. Cytometric bead array (CBA), although more expensive, is considered to be more sensitive and faster than ELISA and very useful for the detection of multiple cytokines in small volumes (Ooi et al. 2006). In this paper, the CBA technique was used to simultaneously evaluate the concentrations of CXCL8, CXCL9, CXCL10, CCL2 and CCL5 in the plasma of TB patients. To our knowledge, this is the first report to evaluate the effects of anti-TB chemotherapy on blood chemokine levels by CBA and to evaluate whether plasma chemokine levels differed between TB patients and BCGvaccinated healthy controls $(\mathrm{HC})$.

This work was approved by the Medical Ethical Committee of the Federal University of Juiz de Fora (174/2006). Patients were recruited from the Respiratory Diseases Division of the Central Public Health Clinic of Juiz de Fora, state of Minas Gerais, Brazil. All patients had sputum-positive bacilloscopy or culture-confirmed disease and had X-ray abnormalities that were consistent with pulmonary TB. AIDS, diabetes, hepatitis, hypertension, pregnancy, malignancies, malnutrition, treatment failures and alcoholism were exclusion criteria. Twentythree patients with active tuberculosis (ATB) (with $\leq 1$ week of treatment) and 20 patients who completed the six-month chemotherapy (TTB group) were evaluated. All patients received conventional treatment for TB (2 months of rifampicin/isoniazid/pyrazinamide followed by 4 months of rifampicin/isoniazid). Most of the patients were male $(63 \%)$ and presented a BCG vaccination scar $(72 \%)$. The age of the patients varied between 13-75 years and the average age was 37 years. Thirty healthy BCG-vaccinated individuals (10 ppd+ and 20 ppd-) with 
no previous history of TB disease were included as HC. The age of the HC varied between 19-30 years and the average age was 24 years. All patients and $\mathrm{HC}$ gave informed consent for blood sampling after written information was provided.

Plasma was separated by density centrifugation on a Ficoll-Plaque Plus (Amersham Biosciences, Uppsala, Sweden). All samples were stored at $-70^{\circ} \mathrm{C}$. The samples were thawed and excess proteins were removed by acid/ salt precipitation. The supernatants were then adjusted for salt content $(0.14 \mathrm{M}$ sodium chloride and $0.01 \mathrm{M}$ sodium phosphate) at $\mathrm{pH} 7.4$ to determine the chemokine concentrations. The CBA technique was used to detect CXCL9, CXCL10, CXCL8, CCL5 and CCL2 using the Human Chemokine-I kit as described by the manufacturer (Becton Dickinson, San Jose, CA, USA). The acquisition and analysis of standards and samples were performed using the FACScalibur flow cytometer and the BD software CBA Isotype Analysis (Becton Dickinson, San Jose, CA, USA).

Data were compared using the Kruskal-Wallis test and are presented as medians. Differences between groups were assessed using Dunn's Multiple Comparison test. A p $<0.05$ was considered statistically significant. Statistical analyses were performed using GraphPad Prism 5.0 software (GraphPad Software Inc, San Diego, CA, USA).

CXCL8, CXCL9 and CXCL10 (Figs 1-3) were higher in patients with ATB compared to both the $\mathrm{HC}$ and TTB $(p<0.05)$. In TTB patients, the levels of CXCL8, CXCL9 and CXCL10 decreased to levels close to those observed in HC. Only CCL2 levels showed no significant difference when the groups of TB patients were compared before and after treatment (Fig. 4). Following six-months of chemotherapy, plasma levels of CCL5 were significantly lower compared to either $\mathrm{HC}$ or ATB (Fig. 5). Similar results were obtained when these chemokines were evaluated in the same group of TB patients $(\mathrm{n}=8-11)$ in a follow-up study before and after the completion of chemotherapy (Supplementary data). In addition, although the $\mathrm{TB}$ and $\mathrm{HC}$ groups were of different mean ages, which could possibly affect serum chemokine levels, there was no correlation between age and chemokine production when the Spearman rank correlation coefficient was used (data not shown).

In this paper, CXCL8, CXCL9 and CXCL10 plasma levels detected in patients with ATB were higher than in BCG-vaccinated HC. Our group showed recently that CXCL9 expression in vitro correlates significantly with enhanced T-cell IFN- $\gamma$ production induced by $M$. tuberculosis-specific antigens (Abramo et al. 2006). We were able to demonstrate the reduction of CXCL8, CXCL9 and CXCL10 after chemotherapy in TB patients for the first time using the CBA technique. Using ELISA, CXCL8 and CXCL9 were reported to be decreased in plasma after anti-TB treatment (Alessandri et al. 2006). In contrast to our results, Alessandri et al. (2006) found no difference in CXCL10 levels between TB patients and control subjects. Our data, however, are consistent with other ELISA studies showing low levels of CXCL10 in the plasma of cured TB patients (Azurri et al. 2005).
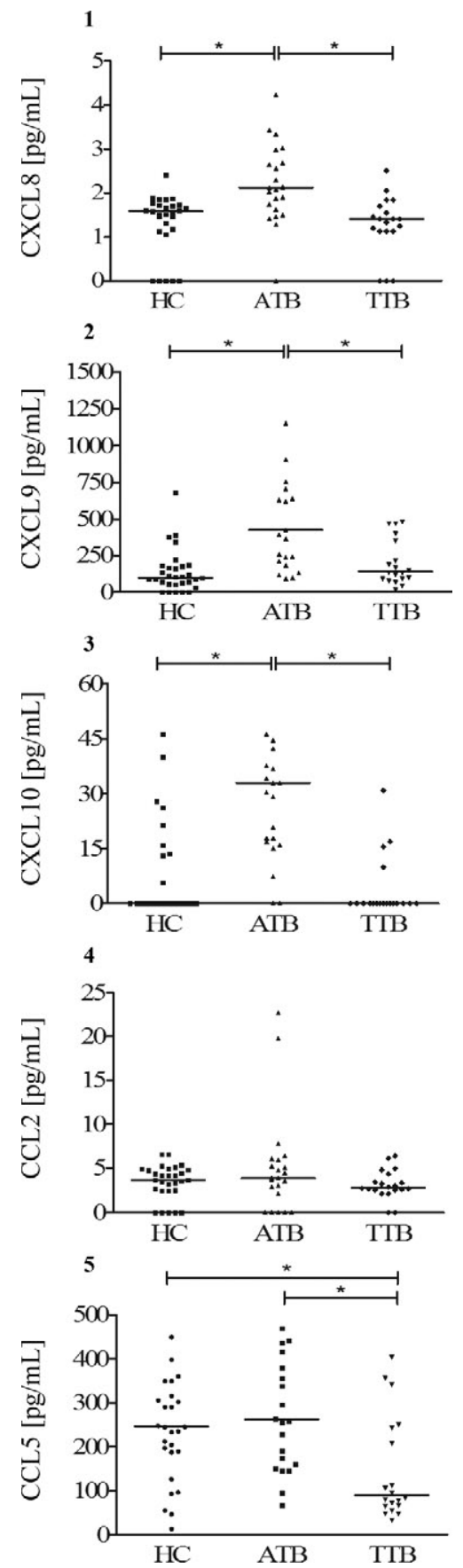

Figs 1-5: CXCL8 (1), CXCL9 (2), CXCL10 (3), CCL2 (4) and CCL5 (5) detected in plasma of patients with active tuberculosis (ATB) $(n=23)$, in patients after completion treatment (TTB) of six months chemotherapy $(\mathrm{n}=20)$ and in plasma of BCG-vaccinated healthy controls $(\mathrm{HC})(\mathrm{n}=30)$ by cytometric bead array. Asterisks means $\mathrm{p}<0.05$.

CCL5 plasma levels were high in the control group. mRNA for CCL5 in spleen cells and in macrophages of guinea pigs increased after vaccination with BCG (Skwor et al. 2006). BCG vaccination and frequent exposure to environmental mycobacteria could in part explain the high CCL5 levels in the plasma of BCG-vaccinated HC from Juiz de Fora, an endemic area of TB in Brazil. In 
addition, there were no significant differences in plasma CCL2 levels between any of the groups studied. Similar findings were obtained by Alessandri et al. (2006), as they demonstrated that levels of CCL2 in the serum or plasma of patients with ATB were not different from the HC. Similarly, high CXCL8 and low CCL2 levels were described in patients with both pulmonary and extrapulmonary ATB compared to $\mathrm{HC}$ from an endemic area (Hasan et al. 2005).

Our findings indicate that $M$. tuberculosis is a strong inducer of CXCL8, CXCL9 and CXCL10. Higher expression of these chemokines could be due to increased numbers of M. tuberculosis bacilli. Alternatively, higher chemokine levels could alter the anti-mycobacterial response, resulting in higher bacterial burden, as high chemokine levels can saturate a receptor and desensitise the cell to the migration signal (Algood et al. 2003). In both situations, it is reasonable to state that anti-mycobacterial chemotherapy leads to diminished levels of the induced chemokines.

In summary, our results show that patients with ATB produce high levels of CXCL8, CXCL9 and CXCL10, which are diminished to control levels after anti-TB chemotherapy. Detection of blood chemokines may provide an important strategy for immunological profiling in TB and for monitoring treatment efficacy.

\section{AKNOWLEDGEMENTS}

To the cooperation of the staff of the Respiratory Diseases Division of the Central Public Health Clinic of Juiz de Fora.

\section{REFERENCES}

Abramo C, Meijgaarden KE, Garcia D, Franken KLMC, Klein MR, Kolk AJ, Oliveira SC, Ottenhoff THM, Teixeira HC 2006. Monokine induced by interferon gamma and IFN-gamma response to a fusion protein of Mycobacterium tuberculosis ESAT-6 and CFP10 in Brazilian tuberculosis patients. Microbes Infect 8: 45-51.

Alessandri AL, Souza AL, Oliveira SC, Macedo GC, Teixeira MM, Teixeira AL 2006. Concentrations of CXCL8, CXCL9 and sT-
NFR1 in plasma of patients with pulmonary tuberculosis undergoing treatment. Inflamm Res 55: 528-533.

Algood HMS, Chan J, Flynn JL 2003. Chemokines and tuberculosis. Cytokine \& Growth Factor Rev 14: 467-477.

Azzurri A, Sow OY, Amedei A, Bah B, Diallo S, Peri G, Benagiano M, D’Elios MM, Mantovani A, Del Prete G 2005. IFN-gammainducible protein 10 and pentraxin 3 plasma levels are tools for monitoring inflammation and disease activity in Mycobacterium tuberculosis infection. Microbes Infect 7: 1-8.

Hasan Z, Zaidi I, Jamil B, Khan MA, Kanji A, Hussain R 2005. Elevated ex vivo monocyte chemotactic protein-1 (CCL2) in pulmonary as compared with extra-pulmonary tuberculosis. $B M C$ Immunol 6: 14-23.

Lu B, Rutledge BJ, Gu L, Fiorillo J, Lukacs NW, Kunkel SL, North R, Gerard C, Rollins BJ 1998. Abnormalities in monocyte recruitment and cytokine expression in monocyte chemoattractant protein 1-deficient mice. J Exp Med 187: 601-608.

Ooi KG, Galatowicz G, Towler HM, Lightman SL, Calder VL 2006. Multiplex cytokine detection versus ELISA for aqueous humor: IL-5, IL-10, and IFN- $\gamma$ profiles in uveitis. Invest Ophthalmol Vis Sci 47: 272-277.

Pokkali S, Das SD 2009. Augmented chemokine levels and chemokine receptor expression on immune cells during pulmonary tuberculosis. Hum Immunol 70: 110-115.

Seiler P, Aichele P, Bandermann S, Hauser AE, Lu B, Gerard NP, Gerard C, Ehlers S, Mollenkopf HJ, Kaufmann SH 2003. Early granuloma formation after aerosol Mycobacterium tuberculosis infection is regulated by neuthophils via CXCR3-signaling chemokines. Eur J Immunol 33: 2676-2686.

Skwor TA, Sedberry AS, Mackie JT, Russell K, Berghman LR, McMurray DN 2006. BCG vaccination of guinea pigs modulates Mycobacterium tuberculosis-induced CCL5 (RANTES) production in vitro and in vivo. Tuberculosis 86: 419-429.

Zhang Y, Broser M, Cohen H, Bodkin M, Law K, Reibman J, Rom WN 1995. Enhanced interleukin-8 release and gene expression in macrophages after exposure to Mycobacterium tuberculosis and its components. J Clin Invest 95: 586-592. 


\section{CXCL8}

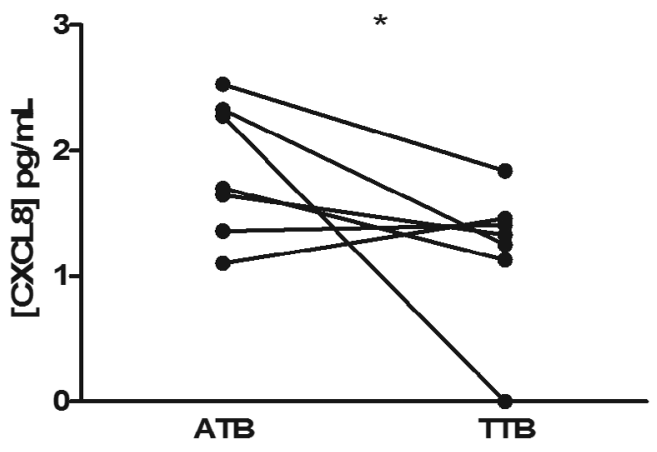

CXCL10

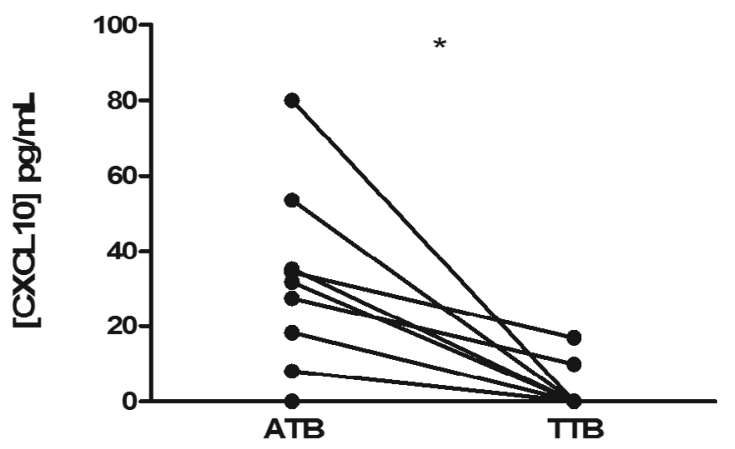

CXCL9
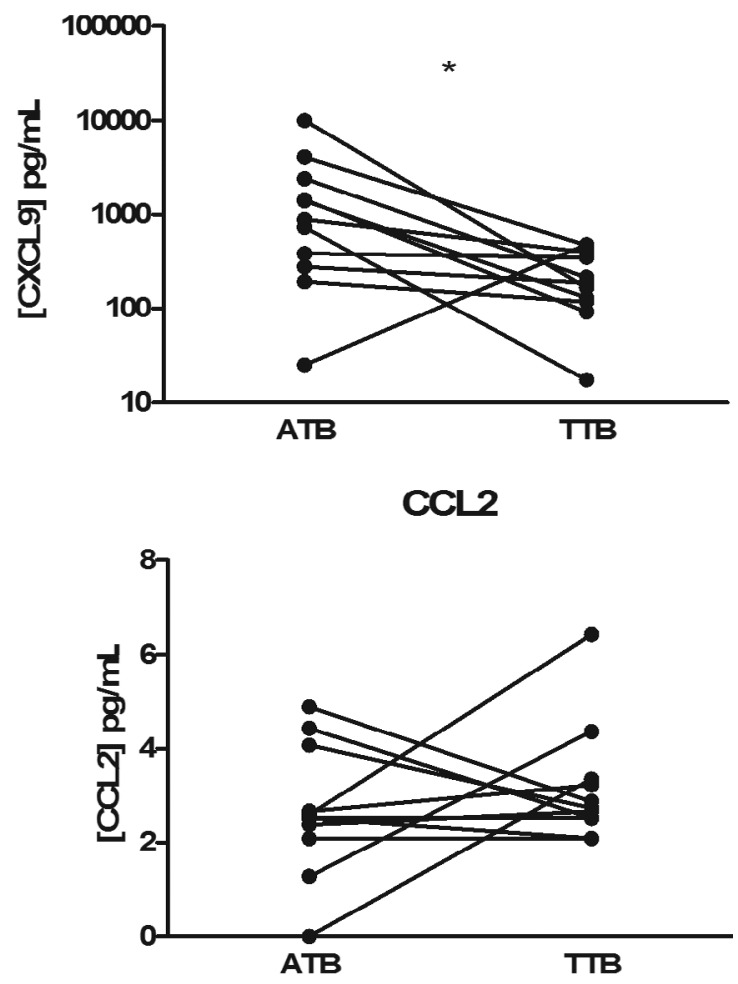

CCL5

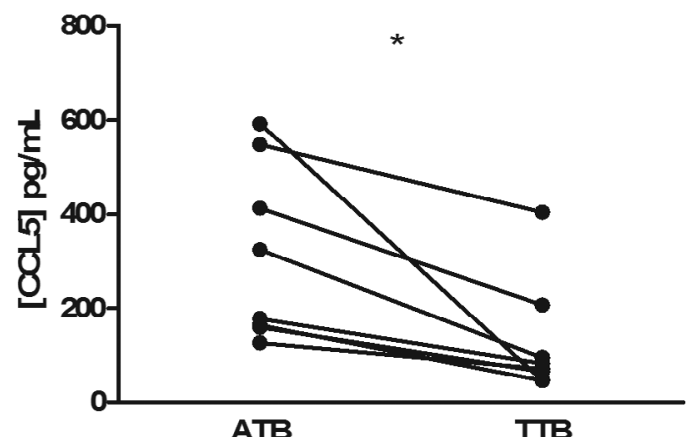

CXCL8, CXCL9, CXCL10, CCL2 and CCL5 plasma levels detected in patients with active pulmonary tuberculosis (ATB) by cytometric bead array. The same group of patients was studied after completion of the six-month chemotherapy (TTB). $n=11$ for CXCL9, CXCL10 and CCL2; $n$ $=8$ for CXCL8 and CCL5. Asterisks means $\mathrm{p}<0.05$ between the ATB and TTB medians (Willcoxon matched pairs test). 